\title{
К БИОЛОГИЧЕСКОИ КОНСТРУКЦИИ ВРЕМЕНИ В ГЕОЛОГИИ
}

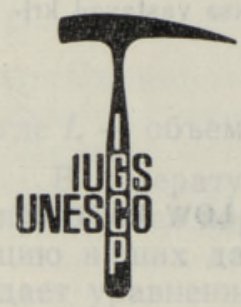

Проект «Экостратиграфия»

\section{1. Постановка задачи}

1.1. Как известно (Косыгин и др., 1974), абсолютное или физическое время может быть определено последовательностью операционных моментов периодического процесса $t_{i}$, для которых принимается допущение $t_{1}-t_{2}=$ $=t_{2}-t_{3}=\ldots=t_{i-1}-t_{i}=\ldots$.

Относительное или геологическое время может быть определено последовательностью таких операционных геологических событий $g_{i}$, которые могут быть упорядочены в кортеж (Шиханович, 1965) $\left\langle g_{1}, \ldots, g_{n}\right\rangle$. Если каждому событию $g_{i}$ соответствует один момент времени $t_{i}$, то должно выполняться отношение порядка $t_{1}<t_{2}<\ldots<t_{n}$.

1.2. Поставим задачу фиксировать распределение палеонтологических объектов (ископаемых остатков древних организмов) относительно шкалы абсолютного времени и создать на этой базе конструкцию биологического времени, которая удовлетворяла бы определению геологического времени и служила инструментом корреляции при биостратиграфических построениях.

\section{2. Модель}

2.1. Допустим, что согласно некоторой биологической концепции множество палеонтологических объектов $A$ разбито на $n$ таксонов $A_{i}$ так, что $A_{i} \cap A_{k}=0, i \neq k ; \cup A_{i}=A$ и $A_{i} \neq 0, i, k=1, \ldots, n$.

Поставим каждому объекту $a \in A$ в соответствие единичный момент абсолютного времени $t$ и пару географических координат $x, y$, фиксирующих аутохтонное захоронение ископаемых остатков.

2.2. В целях упрощения модели введем трехмерное эвклидовое пространство $E(t, x, y)$. При этом не будем рассматривать движение материков, т. е. условие $x, y=f(t)$, а также расстояния $d$ между точками нахождения палеонтологических объектов, т. е. условие $d=f(t, x, y, z)$, где $x, y$ и $z$ определяют геологическое пространство в геоцентрической системе координат (Геология и математика, 1967)..

2.3. Тогда полнозаданное распределение таксона $A_{i}$ будет определяться множеством точек $\{a(t, x, y)\} \in A_{i}$. 


\section{3. Свойства распределения таксонов}

3.1. Хорошо известно, что конфигурация «биологически непрерывного» тела, представленного множеством точек $\{a(t, x, y)\} \in A_{i}$ в пространстве $E$, очень сложнд.

Обозначив моменты времени * $a_{i}=\left\{a\left(t_{\max }, x_{p}, y_{q}\right)\right\} \in A_{i}, \bar{a}_{i}=$ $=\left\{a\left(t_{\min }, x_{p}, y_{q}\right)\right\} \in A_{i}$ и интервал времени (=iocal range) $\vec{a}_{i}=\left[a_{i}, \vec{a}_{i}\right]$, будем считать, что распределение таксона $A_{i}$ таково, что всегда найдутся такие $\overline{\underline{u}}_{i}$, которые не совпадают между собой во времени.

3.2. Значения $\underline{A}_{i}=\max _{x} \underline{a}_{i}$ назовем появлением и $\bar{A}_{i}=\min _{x, y} \bar{a}_{i}-$ вымиранием таксона $A_{i}$. Интервал времени $\underline{A}_{i}=\left[\underline{A}_{i}, \bar{A}_{i}\right]$ соответствует времени существования таксона $A_{i}$ (range).

Теоретически можно допустить, что появления и вымирания исследуемых таксонов не совпадают во времени (Shaw, 1964; Hay, 1972), т. е. $\underline{A}_{i} \neq \underline{A}_{k}, \quad \bar{A}_{i} \neq \bar{A}_{k}, \quad \underline{A}_{i} \neq \bar{A}_{k}, \bar{A}_{i} \neq \underline{A}_{k}$, где $i \neq k$, а также $\underline{A}_{i} \neq \bar{A}_{i}$, где $i$, $\bar{k}=1, \ldots, n$.

3.3. Из опыта известно также, что во временном отношении таксоны могут быть последовательными или одновременными. Уточним это свойство при помощи следующих понятий.

Будем считать два таксона $A_{i}$ и $A_{k}$ последовательными, если их ранговые интервалы $\underline{A}_{i}$ и $\underline{A}_{k}$ не перекрываются во времени, т. е. $\vec{A}_{i} \cap \vec{A}_{k}=0, i \neq k$. Следовательно, таксон $A_{i}$ предшествует таксону $A_{k}$ (или $A_{k}$ следует за $A_{i}$ ) только тогда, когда $\bar{A}_{i}>\underline{A}_{k}$.

Будем называть два таксона $A_{i}$ и $A_{k}$ одновременными, если их ранговые интервалы $\bar{A}_{i}$ и $\bar{A}_{k}$ хотя бы частично перекрываются, т. е. $\overline{\bar{A}}_{i} \cap \bar{A}_{k} \neq 0, i \neq k$.

Будем считать два таксона $A_{i}$ и $A_{k}$ контактирующими, если они последовательны и между ними не имеется ни одного другого таксона, который был бы относительно них последовательным, т. е. имеет место кортеж $\left\langle\underline{A}_{i}, \underline{A}_{h}\right\rangle, i \neq k$.

\section{4. Определения биологического времени}

4.1. Исходя из указанных выше свойств распределения таксонов, имеет смысл выбирать для определения биологического времени только такие $\underline{a}_{i}$ и $\bar{a}_{i}$, которые соответствуют максимальному и минимальному значению времени существования данного таксона, т. е. $\underline{A}_{i}$ и $\bar{A}_{i}$.

4.2. Тогда требуемое биологическое время можно определить последовательностью несовпадающих во времени событий $\underline{A}_{i}$ и $\bar{A}_{i}$, где $i=1, \ldots, n$, т. е. кортежем $\left\langle A_{1}, \ldots, A_{n}\right\rangle$ (напр., Martini, 1970). Число элементов данного кортежа равно $2 N$, где $N$ - число таксонов. Это предел точности определения биологического времени, так как здесь исполь-

* Значение $t=0$ соответствует настоящему моменту; значения $t>0$ увеличиваются в сторону геологического прошлого. 
зованы все возможные кортежи событий $\quad \underline{A}_{i}$ и $\bar{A}_{i}: \quad\left\langle\underline{A}_{i}, \underline{A}_{k}\right\rangle, \quad\left\langle\bar{A}_{i}, \bar{A}_{k}\right\rangle$, $\left\langle\underline{A}_{i}, \bar{A}_{k}\right\rangle, \quad\left\langle\bar{A}_{i}, \underline{A}_{k}\right\rangle$, если $i \neq k$, а также $\left\langle\underline{A}_{i}, \bar{A}_{i}\right\rangle$, если $i, k=1, \ldots, n$.

Однако основанием для данного определения времени служит принятое допущение о несовпадении событий $A_{i}$ и $\bar{A}_{i}$ (см. свойство 3.2 ). Следуя этому определению, принципиально невозможно учесть относительную одновременность появления или вымирания таксонов, имеющую существенное значение в некоторых представлениях о стратиграфических построениях (Халфин, 1974).

4.3. Названный недостаток можно исключить построением модели биологического времени с меньшим числом учитываемых событий, т. е. только на основе свойств 3.1 и 3.3 .

Образуем из множества всех таксонов $A=\left\{A_{i}\right\}$ множество всех упорядоченных пар множества $A$ :

$$
A \times A=\left\{\left\langle A_{1}, A_{1}\right\rangle, \quad\left\langle A_{1}, A_{2}\right\rangle, \ldots,\left\langle A_{n}, A_{n-1}\right\rangle,\left\langle A_{n}, A_{n}\right\rangle\right\} .
$$

Определим подмножества $P, Q$ и $R$ множества $A \times A$ следующим образом:

$$
\begin{aligned}
& \left\langle A_{i}, A_{k}\right\rangle \in P, \text { если } \underline{\bar{A}}_{i} \cap \underline{\bar{A}}_{k} \neq 0 ; \\
& \left\langle A_{i}, A_{k}\right\rangle \in Q, \text { если } \underline{\bar{A}}_{i} \cap \underline{\bar{A}}_{k}=0 \text { и } \underline{\bar{A}}_{i}>\underline{A}_{k} ; \\
& \left\langle A_{i}, A_{k}\right\rangle \in R, \text { если } \overline{\bar{A}}_{i} \cap \underline{A}_{k}=0 \text { и } \underline{\bar{A}}_{i}<\bar{A}_{k} .
\end{aligned}
$$

Удобным способом задания отношений $P, Q$ и $R$ на конечном множестве $A$ является матрица, где одновременность двух таксонов $A_{i}$ и $A_{k}$, т. е. $\left\langle A_{i}, A_{k}\right\rangle \in P$, а также $\left\langle A_{i}, A_{i}\right\rangle \in P$, обозначается знаком « $0 »$, а последовательность - знаками «+», если $\left\langle A_{i}, A_{k}\right\rangle \in R$, и «-», если $\left\langle A_{i}, A_{k}\right\rangle \in Q:$

$$
\begin{aligned}
& A_{1} \quad A_{2} \ldots A_{i} \quad A_{k} \ldots A_{n} \\
& A_{1} \quad 0 \quad 0 \ldots++\ldots+ \\
& A_{2} \quad 0 \quad 0 \ldots++\ldots+ \\
& \text {.................................. } \\
& A_{i}--\ldots 0+\ldots+ \\
& A_{k}--\ldots-0 \ldots+ \\
& A_{n}--\ldots--\ldots 0
\end{aligned}
$$

Отношения $P, Q$ и $R$, выраженные в такой матричной форме, мож• но упорядочить следующим образом:

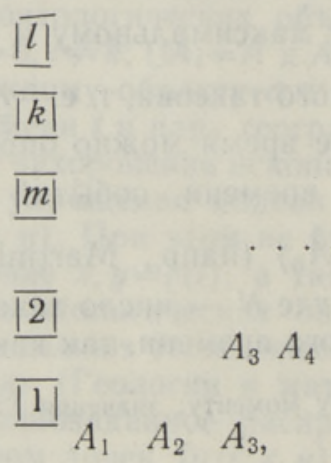


Таблица

\begin{tabular}{|llll|}
\hline $\mathbf{A}$ & & & $\overline{\mathrm{a}}_{4}$ \\
& & & $\mathrm{a}_{4}$ \\
& & $\overline{\mathrm{a}}_{7}$ & $\mathrm{a}_{4}$ \\
& & $\mathrm{a}_{7}$ & $\mathrm{a}_{4}$ \\
& $\overline{\mathrm{a}}_{6}$ & & \\
$\overline{\mathrm{a}}_{8}$ & $\mathrm{a}_{6}$ & & \\
& $\mathrm{a}_{6}$ & $\underline{\mathrm{a}}_{7}$ & \\
& $\mathrm{a}_{6}$ & & \\
& $\underline{\mathrm{a}}_{6}$ & & \\
& & & \\
& $\overline{\mathrm{a}}_{5}$ & & \\
& $\underline{\mathrm{a}}_{5}$ & & \\
& $\overline{\mathrm{a}}_{2}$ & $\overline{\mathrm{a}}_{3}$ & $\mathrm{a}_{4}$ \\
& $\mathrm{a}_{2}$ & $\mathrm{a}_{3}$ & $\mathrm{a}_{4}$ \\
& $\mathrm{a}_{2}$ & & \\
& & & \\
& & & \\
$\bar{a}_{1}$ & $\mathrm{a}_{2}$ & & \\
$\mathrm{a}_{1}$ & $\underline{\mathrm{a}}_{2}$ & & \\
$\mathrm{a}_{1}$ & & & \\
$\mathrm{a}_{1}$ & & & \\
\hline
\end{tabular}

$$
\begin{aligned}
& \begin{array}{lllllllllll}
\text { B } & a_{1} & a_{2} & a_{3} & a_{4} & a_{5} & a_{6} & a_{7} & a_{8} & \Sigma+ & \Sigma-
\end{array} \\
& \mathrm{a}_{1} 00 \mathrm{0}+\mathrm{t}+\mathrm{t}+60 \\
& \begin{array}{lllllllll}
\mathrm{a}_{2} & 0 & 0 & 0 & 0 & + & + & 0
\end{array} \\
& a_{3}-000++++41 \\
& a_{4}-\begin{array}{lllllllll}
0 & 0 & 0 & 0 & 0 & 0 & 0 & 0 & 1
\end{array} \\
& a_{5}---00+++33 \\
& \mathrm{a}_{6}-\ldots-0-0 \quad 0 \quad 0 \quad 0 \quad 0 \quad 4 \\
& \mathrm{a}_{7}-\quad-\quad 0-00000004 \\
& a_{8}-\quad-0-0 \quad 0 \quad 0 \quad 0 \quad 4 \\
& \begin{array}{lllllllllll}
\mathbf{C} & \mathrm{a}_{1} & \mathrm{a}_{2} & \mathrm{a}_{3} & a_{5} & a_{4} & \mathrm{a}_{6} & \mathrm{a}_{7} & \mathrm{a}_{8} & \boldsymbol{\Sigma}+ & \boldsymbol{\Sigma}-
\end{array} \\
& a_{1} 00++++++60 \\
& \mathrm{a}_{2} \quad 000+0+++40 \\
& \mathrm{a}_{3}-00+0+++41 \\
& \mathrm{a}_{5}---00+++33 \\
& a_{4}-0 \begin{array}{lllllllll}
0 & 0 & 0 & 0 & 0 & 0 & 0 & 0 & 1
\end{array}
\end{aligned}
$$

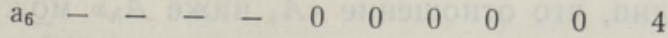

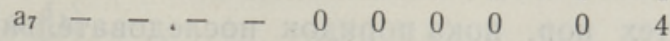

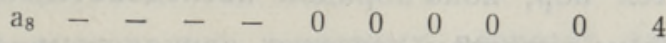

$$
\begin{aligned}
& \text { D }
\end{aligned}
$$

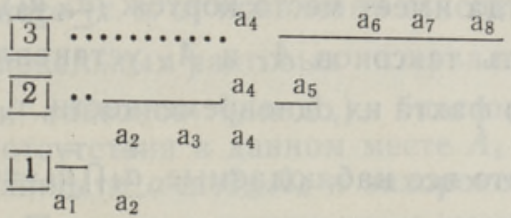

Любое единичное распределение $\mathbf{A}$, где $a_{i} \in A_{i}, i=1, \ldots, 8$, может быть представлено в форме В, C и D. В матрище D контактирующие таксоны разделены сплошной линией; в матрице А вертикальные расстояния между $a_{i}$ соответствуют расстояниям их находок в разрезе.

где в каждой $j$-й строке (начиная с первой снизу) помещены символы таксонов, являющихся одновременными для данного уровня, а в $j+1$-й строке - сначала символы таксонов, одновременных с указанными в строке $j$, а затем - контактирующих с ними. Слева (в квадратах) приведена нумерация контактов (см. таблицу).

В этом случае биологическое время может быть определено последовательностью контактов, т. е. кортежем $\langle\underline{\overline{|1|}}, \ldots, \overline{|l|}\rangle$.

\section{5. Техника построения модели}

5.1. Согласно обоим определениям биологического времени (4.2 и 4.3) необходимо из всех наблюдаемых в частных разрезах событий $\underline{a}_{i}$ и $\bar{a}_{i}$ выявить те, которые соответствуют появлению $\underline{A}_{i}$ и вымиранию $\bar{A}_{i}$ данного таксона.

5.2. Допустим, что направление времени в смысле $t_{i}>t_{k}$ во всех изучаемых разрезах известно. Тогда поставленная задача решается изучением последовательности событий, наблюдаемых в этих разрезах. Исходим при этом из ограничения, обеспечивающего непротиворечивость понятия об относительном времени: «...для любой пары признаков 
$\alpha$ и $\beta \ldots$ отношения между телами, обладающими этими признаками, должны быть одинаковыми во всех разрезах - либо $\alpha$ везде выше $\beta$, либо наоборот.» (Косыгин и др., 1974, с. 48).

5.3. Модель биологического времени по 4.2 , принятая в вероятностной стратиграфии (Нау, 1972), не удовлетворяет вышеприведенному ограничению, так как она допускает возможность принятия некоторой последовательности событий при наличии редких наблюдений последовательностей противоположного направления.

5.4. Модель биологического времени по 4.3 может быть построена на базе следующих исходных наблюдений: имеется конечное число частных разрезов с известным направлением времени, после опробования которых известно, что имеют место аутохтонные распределения изученных таксонов $\bar{a}_{i}(x, y)$. Искомые события $A_{i}$ и $\bar{A}_{i}$ заданы тогда, когда известно множество $A \times A$. Для определения последнего достаточно установить последовательность или одновременность таксонов $A_{i}$ по всем наблюдаемым $\bar{a}_{i}(x, y)$.

Очевидно, что отношение « $A_{i}$ ниже $A_{k}$ можно считать действительным до тех пор, пока порядок последовательных событий $\underline{\bar{a}}_{i}$ и $\underline{\bar{a}}_{k}$ по всем частным разрезам остается одинаковым, т. е. если все $\bar{a}_{i}(x, y) \cap$ $\cap \bar{a}_{k}(x, y)=0$ и всегда имеет место кортеж $\left\langle\bar{a}_{i}, \bar{a}_{k}\right\rangle$, то $A_{i} Q A_{k}$ или $\bar{A}_{i} R A_{k}$.

Одновременность таксонов $A_{i}$ и $A_{k}$ устанавливается при обнаружении хотя бы одного факта их одновременности, т. е. $\overline{\bar{a}}_{i}(x, y) \cap \underline{\bar{a}}_{k}(x, y) \neq 0$, или при условии, что все наблюдаемые $\underline{\bar{a}}_{i} \cap \underline{\bar{a}}_{k}=0$, но хотя бы один раз встречается противоположный порядок $\left\langle\bar{a}_{i}, \bar{a}_{k}\right\rangle$ и $\left\langle\underline{\underline{a}}_{k}, \bar{a}_{i}\right\rangle$.

Таким образом, задача сводится к составлению матриц $\bar{a}_{i} \times \bar{a}_{i}$, отражающих отношения $\bar{a}_{i} P \bar{a}_{k}, \bar{a}_{i} R \bar{a}_{k}$ и $\bar{a}_{i} Q \bar{a}_{k}$ по каждому разрезу, и «суммированию» их по одноименным элементам в матрицу $A \times A$ согласно следующим правилам: + и + равно $+;-$ и - равно $-; 0$ и 0 равно $0 ;+$ и 0 равно $0 ;-$ и 0 равно $0 ;+$ и - равно 0.

Частные разрезы охватывают различные интервалы времени. Вследствие этого (см. также пункт 7) некоторые отношения таксонов в матрице $A \times A$ остаются нераскрытыми. Часть «пустых» ячеек матрицы $A \times A$ может быть заполнена в соответствии с правилом: если $\vec{A}_{i}>\underline{A}_{k}$ и $\bar{A}_{k}>\underline{A}_{j}$, то $A_{j}$ является последовательным относительно $A_{i}$.

Если расположить строки (и столбцы) этой матрицы в порядке уменьшения числа ячеек со знаком « $+»$ и увеличения числа ячеек со знаком «-» на каждой строке, то порядок строк матрицы $A \times A$ укажет последовательность таксонов во времени.

Если все ячейки матрицы $A \times A$ заполнены и выявленные отношения таксонов соответствуют действительности **, то перевод упорядоченной матрицы $A \times A$ в более наглядную форму (см. с. 138) для выявления контактов осуществляется однозначно ***. Если при этом возникнут противоречия, то требуется дальнейшее изучение материала (см. пункт 7).

** Отметим, что в настоящее время соответствующего формального критерия не существует.

*** Вывод следует из опыта и требует строгого математического доказательства. 


\section{6. Корреляция}

6.1. Под корреляцией частных разрезов понимается (Стратиграфия и математика, 1974) либо установление фаунистической непрерывности (=continuity, Нау, 1972), либо установление возрастных отношений или синхронизации (=correlation s. str., Наy, 1972) геологических тел (Косыгин й др., 1964), представленных в частных разрезах. Корреляция на основе биологического времени относится к последнему из названных подходов.

6.2. Пусть биологическое время $\langle\overline{|l|}, \ldots, \overline{|k|}\rangle$ получено обобщением распределения таксонов по $s$ частным разрезам. Тогда только для этих разрезов действительна следующая процедура корреляции: если последовательность событий $\bar{a}_{i}>\underline{a}_{k}$, наблюдаемая по частным разрезам, совпадает с контактом $\bar{A}_{i}>\underline{A}_{k}$, определяемым биологическим временем, то между точками нахождения $\vec{a}_{i}$ и $a_{k}$ в частных разрезах остается момент времени $\overline{|k|}=\left\langle\bar{A}_{i}, \bar{A}_{k}\right\rangle$.

Расстояние между точками $\bar{a}_{i}$ и $a_{k}$ в частных разрезах зависит от многих факторов: временного (т. е. от появления таксона $A_{k}$ и вымирания $A_{i}$ ), экологического (от локальных ранговых интервалов $\underline{\bar{a}}_{i}, \bar{a}_{k}$, а также от частоты встречаемости таксонов $A_{i}$ и $A_{k}$ ), биогеографического (от первичного наличия или отсутствия в данном месте $A_{i}$ и $A_{k}$ ), тафономического (наличия и сохранности $a \in A_{i}, A_{k}$ в захоронениях) и, конечно, от частоты опробования. Поэтому расположение момента времени $\overline{|k|}$ в частных разрезах устанавливается обыкновенно в пределах некоторого интервала, превышающего минимальное расстояние между точками $\bar{a}_{i}$ и $a_{k}$, соответствующее нахождению $\bar{a}_{i}$ и $\underline{a}_{k}$ в двух непосредственно последовательных пробах. Назовем такие интервалы интервалами неопределенности (см. рисунок). Эти интервалы могут быть сужены различными экстраполяциями распределения таксона $A_{i}$ выше точки $\vec{a}_{i}$ и ниже точки $a_{i}$ (напр., до соответствующих границ литотела, содержащего таксон $A_{i}$ ), но подобные экстраполяции все же в большинстве случаев не дают возможности идентифицировать момент $\overline{k \mid}$ по одной точке разреза.

Следовательно, имеет смысл расчленить все коррелируемые разрезы по учитываемым находкам $a_{i}$ и $\bar{a}_{i}$ на слои $S_{i, j}$, где $i-\overline{\text { номер }}$ частного разреза и $j-$

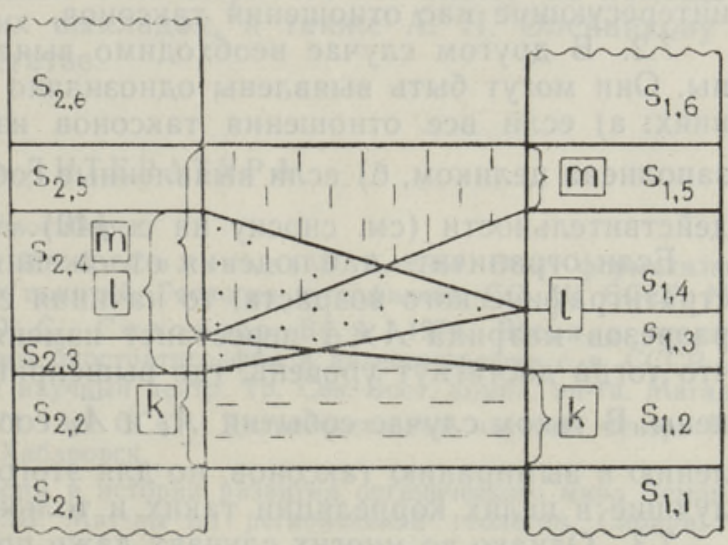

Расчленение и корреляция двух разрезов $S_{1}$ и $S_{2}$ при помощи трех контактов |k|, $\mid \frac{|l|}{\mid \overline{|m|}}$. 
номер слоя в этом разрезе. По отношению к моменту времени $\underline{|k|}$ слои $S_{i . j}$ могут быть «старше» $\left(S_{i, j}>\overline{|k|}\right)$, «моложе» $\left(S_{i, j}<\overline{|k|}\right)$ либо «и старше и моложе» $\left(S_{i, j}<>\overline{|k|}\right)$.

В качестве примера приведем в этой форме корреляцию разрезов, показанных нна рисунке.

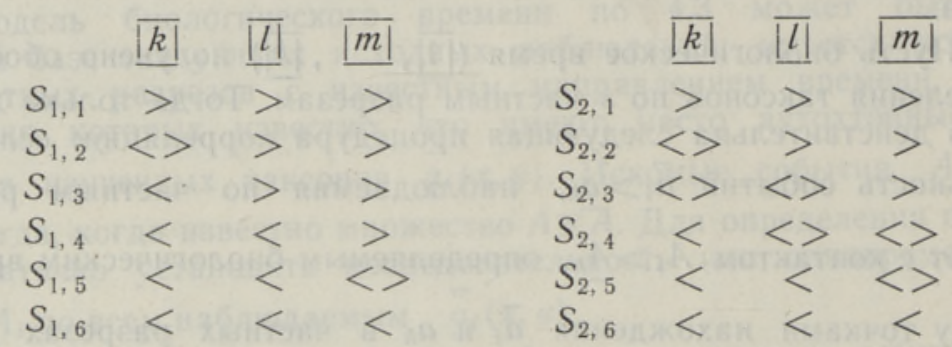

6.3. Достоверность корреляции по биологическому времени зависит от числа и различных взаимоотношений наблюдений. Например, нужные вероятности об определенной последовательности двух контактирующих таксонов $\left\langle\bar{A}_{i}, \bar{A}_{k}\right\rangle$ по числу наблюдений $\left\langle\bar{a}_{i}, \bar{a}_{k}\right\rangle$ можно найти из имеющихся в литературе таблиц (Нау, 1972, табл. 1). Однако рассмотрение этого круга вопросов не входит в задачи данной статьи.

\section{7. Затруднения}

7.1. Обобщение распределения таксонов в частных разрезах для построения модели биологического времени может быть истолковано как самостоятельная задача и как получение инструмента для биостратиграфических корреляций.

В первом случае, когда конечный результат может быть представлен в форме упорядоченной матрицы $A \times A$, особых затруднений не возникает. Такая матрица может либо полностью, либо частично раскрыть интересующие нас отношения таксонов.

7.2. В другом случае необходимо выяснить контактирующие таксоны. Они могут быть выявлены однозначно и полностью при двух условиях: а) если все отношения таксонов известны, т. е. матрица $A \times A$ заполнена целиком, б) если выявленные события $A_{i}$ и $\bar{A}_{i}$ соответствуют действительности (см. сноску на с. 140).

Если ограничить наблюдения отложениями определенного региона и стратиграфического возраста, то начиная с некоторого числа изученных разрезов матрица $A \times A$ перестанет изменяться. Естественно полагать, что тогда достигнут уровень, где вышеприведенные требования выполнены. В таком случае события $A_{i}$ и $\bar{A}_{i}$ соответствуют локальному появлению и вымиранию таксонов, но для этого регнона и возраста они нанлучшие в целях корреляции таких и только таких отложений.

7.3. Однако во многих случаях даже при таком подходе невозможно увеличить число наблюдений до требуемого уровня. Это касается, например, отложений определенного региона и стратиграфического возраста, образовавшихся в условиях сложной фациальной зональности. В таком 
- $2 x+4$

случае достоверность корреляции увеличивается, если моделировать биологическое время для каждой фациальной зоны отдельно. Относительно матрицы $A \times A$ это будет означать составление таких подматриц $A^{\prime} \times A^{\prime}$, где $A^{\prime}$ - экологически эквивалентные таксоны различного стратиграфического возраста. Другими словами, в целях повышения достоверности корреляции необходимо знать экологическую структуру данных таксонов (напр., характер сообществ) .

Последняя задача слишком самостоятельна, чтобы быть решенной в пределах данной статьи, но отметим, что в принципе решение ее требует наличия представлений о временных отношениях вмещающих отложени: (cм. Rubel, 1970). Предположим, что даже неоднозначно выявленные контакты $\left\langle\bar{A}_{i}, \bar{A}_{k}\right\rangle$ уже дают достаточный инструмент для грубого выявления сообществ. В этом случае, если модель биологического времени, полученная на основании хотя бы приближенно выделенных сообществ, позволяет уточнить корреляцию разрезов, имеет смысл повторить предлагаемую процедуру.

Необходимо, однако; отметить, что повышение достоверности корреляции разрезов внутри одной фациальной зоны еще не решает вопроса о взаимной увязке биологического времени различных фациальных зон.

\section{Заключение}

Биостратиграфической классификации частных разрезов процедурно предшествует их корреляция (Стратиграфия и математика, 1974). Если последняя основывается только на данных распределения таксонов, то первой задачей (процедурой) является обобщение распределения таксонов по многим разрезам в шкалу, показывающую временные отношения этих таксонов.

Шкала биологического времени выражается последовательностью биостратиграфических событий. Если известна такая последовательность, то только контакты определенных событий, а не события сами, показывают однозначно временные моменты или интервалы расположения этих моментов в коррелируемых частных разрезах.

Автор выражает искреннюю благодарность С. Ульму за помощь в оформлении математических выкладок, а также А. Н. Олейникову за критические замечания к статье.

\section{Л И ТЕ Р А Т Р А}

Геология и математика. 1967. Новосибирск.

Косыгин Ю. А., Воронин Ю. А., Соловьев В. А. 1964. Опыт формализации некоторых тектонических понятий. Геология и геофизика, СО АН СССР, № 1.

Косыгин Ю. А., Салин Ю. С., Соловьев В. А. 1974. Время в геологии. В сб.: Основные проблемы биостратиграфии и палеогеографии с.-в. СССР. АН СССР. Дальневосточный научный центр. Тр. Сев.-Вост. компл, нн-та. Магадан.

Стратиграфия и математика. 1974. АН СССР. Дальневосточный научный центр. Ин-т тектоники, и геофизики. Хабаровск.

Х ал фин Л. Л. 1974. Смена форм в истории развития органического мира Земли фактическая основа МСШ. Мат-лы по региональной геологии Сибири. Тр. СНИИГГИМС, вып. 173.

Ш и х а н о в и Ю. А. 1965. Введение в современную математику. М.

H a y, W. W. 1972. Probabilistic stratigraphy. Eclogae geol. Helv., 65/2.

Martini E. 1970. Standard Paleogene Calcareous Nannoplankton Zonation. Nature, 226. 
Rubel M. 1970. On the distribution of brachiopods in the lowermost Llandovery of Estonia. ENSV TA Toimet., Keem. Geol., 19, Nr. 1.

Sh aw A. B. 1964. Time in stratigraphy. McGraw-Hill Book Co., New York.

\author{
Ннститут геологии \\ Академии наук Эстонской ССР \\ Поступила в редакцию \\ $13 /$ II 1975
}

M. RUBEL

\title{
AJA BIOLOOGILISEST KONSTRUEERIMISEST GEOLOOGIAS
}

Järjestikuste, üheaegsete ja kontakteeruvate taksonite abil vaadeldakse suhtelise ajaskaala konstrueerimist geoloogias ja selle kasutamist läbilöigete korreleerimisel.

\section{RUBEL}

\section{ON BIOLOGICAL CONSTRUCTION OF TIME IN GEOLOGY}

Two taxa are defined as sequential in time if their total ranges do not coincide.

Two taxa are defined as contemporaneous if their total ranges coincide even if only partially.

Two taxa are defined as contacting if they are sequential and between their total ranges there are no total ranges of any other taxa that are sequential with regard to them.

It is obvious that any taxon has at least one contact.

A sequence of the contacts between taxa is considered as a biological construction of the geological (relative) time scale.

A technology is proposed for establishing the contacts between taxa on the basis of local ranges of taxa, and the use of such a time scale in correlation, and possible difficulties in complicated situations are discussed. 